\title{
Model-Based Verification of Automotive Control Software
}

\author{
Rance Cleaveland \\ Department of Computer Science and \\ Fraunhofer USA Center for Experimental Software Engineering, \\ University of Maryland, USA
}

\begin{abstract}
This talk will report on the use of an approach, called Instrumentation Based Verification, for checking the correctness of models of control software given in Simulink ${ }^{\circledR}$ and Stateflow ${ }^{\circledR}$. In IBV, engineers formalize requirements as so-called monitor models, whose purpose is to search executions of the main controller model for violations of required behavior. Testing is then performed on the instrumented controller model in order to check for the possibility of deviations between controller and requirements. Tools such as Reactis ${ }^{\circledR}$ provide automated support for conducting these activities, and the technique has attracted interest in automotive, aerospace and medical-device settings. The presentation will first review model-based development and IBV and their industrial motivations. It will then report on a project between the Fraunhofer Center for Experimental Software Engineering and a major automotive supplier on using IBV to verify models of an exterior-lighting control system.
\end{abstract}

